\title{
A cascaded pushing displacement estimation approach for hydraulic powered roof support based on multi-segmental Kalman filter
}

\author{
Lin Zhang*, Shang Feng, Minzhou Luo and Aimin Ji
}

To tackle the problem of non-reusability of the magnetostrictive displacement sensor which is embedded in a pushing hydraulic cylinder, and improve the accuracy of pushing displacement sensing for hydraulic powered roof support, the compact self-contained inertial sensor is utilized in pushing displacement measurement. The motion characteristics of pushing operation are re-considered, and multi-segmental Kalman filter (MS-KF) is proposed based on the motion characteristics. A cascaded framework is constructed for pushing displacement estimation, and key technologies such as orientation estimation, segmental recognition and MS-KF implementation are demonstrated. The experiment is elaborated and experimental results show that the proposed approach significantly reduces the cumulative error and proves to be practical and valuable.

Keywords: Inertial sensor, hydraulic support, pushing displacement, Kalman filter.

As one of the most intelligent equipment in fully mechanized coal mining working face, the hydraulic powered roof support (HPRS) works cooperatively with cutting machine (also known as shearer or mining machine) and scraper chain conveyor. Pushing link, which physically connects the scraper chain conveyor and hydraulic support, is utilized to push the scraper chain conveyor forward or backward. During each working cycle, HPRS will cooperatively push the corresponding part of scraper conveyor from the current position to the next. HPRS will advance forward to be in alignment with the same baseline. During the periodic pushing and advancing, pushing displacement of every hydraulic support is the key metric to percept the smoothness of scraper chain conveyor. Due to poor work environment, different geological conditions and loss reduction of hydraulic emulsion system, there are still challenges to measure pushing displacement at present.

As a traditional solution, magnetostrictive displacement sensor (MDS) is utilized to measure pushing displacement through rough estimation. To protect the

\footnotetext{
Lin Zhang is in the School of Mechanical and Electrical Engineering, Yangtze Normal University, Chongqing, 408100, China; Minzhou Luo and Aimin Ji are in the College of Mechanical \& Electrical Engineering, Hohai University, Changzhou 213022, China; Shang Feng is in the School of Computer Science and Technology, Harbin Institute of Technology, Harbin 150001, China; Lin Zhang and Minzhou Luo are also in the Jiangsu Key Laboratory of Special Robot Technology, Changzhou 213022, China

*For correspondence. (e-mail: linzhang@yznu.edu.cn)
}

sensor from being damaged when exposed to complicated working space and to improve reusability, MDS has to be embedded into the pushing hydraulic cylinder (PHC). However, it does not guarantee sensing accuracy. The actual pushing displacement is the moving distance of pushing link, while sensing displacement is the length of moving piston, which means there is a deviation between actual pushing displacement and sensing displacement. Thus, pushing displacement must be obtained via simply trigonometry operator or must directly use sensing displacement as an approximate value. However, there are some drawbacks in this approach. First, MDS of hydraulic support is non-reusable due to cumbersome mechanical structure, unmaintainable installation of sensor, and harsh underground work environment. As a consequence, this approach will not work once the MDS is broken, and the broken MDS cannot be replaced until the whole mining process is finished. Secondly, if pushing distance is indirectly approximated via pushing length of PHC, obviously, the approximation error is inevitable. Finally, if pushing distance is calculated via trigonometry transformation, then the calculation error may vary significantly due to different roughness of the floor. Thus, sensing error of pushing displacement may be rather large.

Aiming at the above problems, the compact selfcontained inertial sensor provides potential approach for pushing displacement measurement. In order to reduce estimation error, a cascaded pushing displacement estimation framework based on multi-segmental Kalman 
filter (MS-KF) is proposed. Key technologies of the proposed framework, such as orientation estimation, segmental recognition and $\mathrm{MS}-\mathrm{KF}$ implementation are demonstrated as well.

\section{Literature review}

Recent research works relevant to this study focus on three topics: displacement measurement, inertial sensing approach and Kalman filter. In this section, we list and summarize the related literature.

\section{Displacement measurement}

Since displacement is valuable information for analysing structural behaviour, motion characteristics and mechanical properties, displacement measurement has been applied in various science scenarios, structure engineering ${ }^{1}$, robotics $^{2}$, and medical science ${ }^{3}$, etc. Among these, the most applicable field is structure engineering. Structure health monitoring applications all begin with measuring the dynamic response of structures, but displacement measurement has been limited by the difficulty in requiring a fixed reference point, high cost and low accuracy. Yoon et al. ${ }^{4}$, presented a framework to achieve absolute displacement of a structure with a video taken from an unmanned aerial system using a three-step phased approach. Aimed at long span bridge structures, Soman et $a l . .^{5}$ presented a feature extraction-level data fusion-based damage isolation strategy using multi-metric sensing; both strain and displacement data were combined after performing coordinate transformation. Considering the disadvantages of traditional displacement sensors such as instrumentation cost, installation efforts and measurement capacity in terms of frequency range and spatial resolution, more comprehensive works from the perspective of vision-based displacement measurement have been reviewed by $\mathrm{Xu}$ and Brownjohn ${ }^{6}$. In Europe, the displacement estimation framework is a key part of forestry unmanned aerial vehicles (UAVs); both aerial imagery and GPS information can be utilized for post-harvest soil displacement estimation ${ }^{7}$. In order to estimate soil displacement from timber extraction trails in steep terrain, an unmanned aircraft is used for 3D scanning to generate a detailed post-harvest terrain model ${ }^{8}$. Displacement measurement is widely researched in materials science as well Rizzello et al. focused on self-sensing of displacement for dielectric elastomers, and applied the estimated displacement in closed-loop control ${ }^{9-11}$. Tessler et al. ${ }^{12}$, proposed inverse finite element method to reconstruct the displacement field of a shell structure which undergoes large deformations using discreet strain measurements as the prescribed data. Besides, displacement measurement is also a great challenge for tissue motion estimation ${ }^{13}$, ultrasound elastography ${ }^{14}$, electrode displacement elastography ${ }^{15}$, etc.

\section{Inertial sensing approach}

There are other displacement sensors such as wheel odometer, INS, GPS/GNSS, ultrasonic sensor, laser sensor and optical camera (Table 1$)^{16}$. However, these sensors are difficult to use due to complicated working conditions. On the one hand, the measuring area, which can be used for sensor mounting, will be occupied frequently by human beings, because this is the only available area for the operators. On the other hand, scattered coal block will fly into this area occasionally. Thus, this area is totally exposed to danger, both for the sensors and human beings. Thus, inertial sensors could be appropriate for displacement sensing in this case. As described earlier, the inertial sensor is suitable for this scenario due to the limited working space. The sensor is frequently used for navigation $^{17}$, in robotics $^{18}$ and motion tracking ${ }^{19}$. In order to estimate the full state of the flexible joint manipulators, a nonlinear observer under the Lipschitz condition of nonlinear stiffness is proposed and algebraic Riccati equations are applied to choose the gain of the proposed observer ${ }^{20}$. Comparative experimental results verify the effectiveness of the proposed method. For more complicated and precise requirement, simultaneous localization and mapping (SLAM) and laser scan matcher (LSM) can be improved with the use of inertia sensor and kinematic velocity information ${ }^{21}$. Inertial sensors provide a possible solution since they are self-contained, relatively low cost and can be easily mounted on the machine structure. While the inherent limitation accumulated growth of error, with time, measured velocity and position need more efficient and precise process models or algorithms ${ }^{22}$. To achieve this goal, researchers use other compensate sensors to remove the drift during long-term measurement. Inertial sensors, ultrasonic sensors and magnetometers are combined together to estimate motion state using the extended Kalman filter for data fusion ${ }^{23}$. The results show that the accumulated errors of inertial sensors are reduced by ultrasonic sensors and magnetometers, and extended Kalman filter (EKF)-improves the accuracy of orientation and position measurements.

\section{Kalman filter}

The inertial sensor provides a potential approach for pushing displacement estimation due to its advantages such as lightweight, low power consumption, portability and being self-contained. Although it is successfully used in many fields, displacement estimation based on inertial sensor is still a great challenge due to the cumulative errors $^{24}$. Thus, practical usability of the inertial sensor for displacement estimation requires better signal processing algorithms before it can be used in extreme working condition. As a state-space model-based linear filter ${ }^{25}$, the Kalman filter is frequently used in inertial information 
GENERAL ARTICLES

Table 1. Comparison of commonly used sensors

\begin{tabular}{|c|c|c|}
\hline Sensor/technology & Merits & Demerits \\
\hline Wheel odometry & $\begin{array}{l}\text { Simple computation, short-term accuracy, high } \\
\text { sampling rate and low-cost solution }\end{array}$ & $\begin{array}{l}\text { Position drift } \\
\text { Error accumulation over time }\end{array}$ \\
\hline INS & $\begin{array}{l}\text { Provides both position and orientation. } \\
\text { Not subject to interference outages }\end{array}$ & $\begin{array}{l}\text { Position drift } \\
\text { Long-term drift errors }\end{array}$ \\
\hline GPS/GNSS & $\begin{array}{l}\text { Provides absolute position with known value of } \\
\text { error, No error accumulation over time }\end{array}$ & $\begin{array}{l}\text { Unavailable in indoor, underwater, underground } \\
\text { and in closed areas }\end{array}$ \\
\hline Ultrasonic sensor & $\begin{array}{l}\text { Provides a scalar displacement measurement from } \\
\text { sensor to object. Also, provides inexpensive } \\
\text { solution }\end{array}$ & $\begin{array}{l}\text { Reflection of signal wave } \\
\text { Signal interference } \\
\text { Low angular resolution and scan rate }\end{array}$ \\
\hline Laser sensor & $\begin{array}{l}\text { High accuracy and scan rate. Provides the distance } \\
\text { to a single point or an array of distances }\end{array}$ & $\begin{array}{l}\text { Reflection of signal wave } \\
\text { Expensive solution }\end{array}$ \\
\hline Optical camera & $\begin{array}{l}\text { Images store huge meaningful information. } \\
\text { Provides high localization accuracy and } \\
\text { inexpensive solution }\end{array}$ & $\begin{array}{l}\text { Requires image-processing and data extraction } \\
\text { techniques } \\
\text { High computational cost }\end{array}$ \\
\hline
\end{tabular}

processing with uncertainty problems ${ }^{26}$, including position estimation ${ }^{27}$ as well as rotation estimation ${ }^{28}$. For nonlinear problems, researchers also proposed some mutation of a Kalman filter, such as extended Kalman filter ${ }^{29}$, unscented Kalman filter $^{30}$, etc. Recently, Kalman filter has also been used in intelligent computation and information fusion through distributed or parallel approach $^{31,32}$. In addition, Kalman filter contributes to magnetometer calibration ${ }^{33}$, moving object tracking ${ }^{34}$, and satellite attitude determination ${ }^{35}$. However, the estimation performance of Kalman filter is greatly influenced by the selection of processing noise covariance $Q$ and observation noise covariance $R^{36}$. Thus, tuning method of the covariance should be applied to obtain better estimation performance. Optimization algorithms play an important role for tuning parameters of Kalman filter. Recently, some interesting and efficient nature-inspired optimization algorithms have been proposed for parameter tuning. In 2012, krill herd (KH) was proposed by Gandomi and Alavi ${ }^{37}$. Based on the innovation, improved $\mathrm{KH}$ algorithms are successively being developed ${ }^{38,39}$. These algorithms are efficient and flexible, and can be enhanced either by internal improvement, such as introducing new migration operator ${ }^{40}$ and new information exchange process $^{41}$, or by combining other algorithms together, such as quantum-behaved particle swarm algorithms ${ }^{42}$ and cuckoo search algorithms ${ }^{43}$. Monarch butterfly optimization $(\mathrm{MBO})^{44}$ and $\mathrm{MBO}$-based algorithms ${ }^{45}$ are recently developed meta-heuristic algorithms which simulate the migration of monarch butterflies. Similar algorithms like moth search algorithm ${ }^{46}$, earthworm optimization algorithm $^{47}$, cuckoo search algorithm ${ }^{48}$, bat algorithm ${ }^{49}$ and firefly algorithm ${ }^{50}$ also simulate the natural processes and contribute to intelligent optimization algorithms.

\section{Discussion}

Although the above-mentioned studies are highly valuable, the problem of pushing displacement estimation on mining face has rarely been researched due to existing challenges regarding measurement approach in practical applications. Here, we propose a cascaded displacement estimation approach to estimate the pushing length of PHC without the use of non-reusable MDS. We also combine this approach with multi-segmental Kalman filter to estimate pushing displacement in different signal segments.

\section{Proposed estimation approach}

\section{Description of pushing operation}

Generally, movement of hydraulic support contains two parts: pushing scraper chain conveyor forward (pushing) and advancing itself forward (advancing). The combination of pushing and advancing constitutes a periodic movement. First, hydraulic support utilizes pushing link to push the scraper chain conveyor forward (hydraulic support body is motionless). Then the pushing link will pull the whole body of hydraulic support forward (scraper chain conveyor is motionless). With such a periodic movement, the chain conveyor can be pushed forward step by step. The translation along the $y$-axis of the sensor frame of reference is the main feature of the pushing movement. Due to the roughfloor, rotations around $x-, y$ and $z$-axis will cause some acceleration fluctuations. The analysis is based on the following hypothesis: (i) The density of emulsified liquid is stable and inner diameter of the pipeline is constant. (ii) All hydraulic supports are in good condition with constant flow rate; there is no 
leakage of any emulsified liquid. (iii) Hydraulic pump provides enough pressure to pushing and advancing.

Hydraulic support extends the piston out or withdraws the piston back while pushing; both acceleration and angular rate show significant response to movement. The acceleration shows an increase in the beginning and decrease at the end, while it will present a totally inverse result when advancing. However, their magnitudes are different due to the fact that PHC with floating piston outputs different force magnitude when extending piston out or withdrawing piston back, which leads to varying velocity between pushing and advancing. The curve of displacement seems to be a tilt trapezoid.

Since flow rate outlet of the hydraulic pump for PHC is constant, based on the listed hypnosis, moving velocity of the piston should be constant as well. However, when pushing and advancing at the starting point, the acceleration fluctuates significantly and increases rapidly to an excepted velocity. The velocity should be constant until it reaches the end point according to hypotheses (2) and (3). The angular rate also presents similar features, which can be aid for recognition of pushing and advancing. Based on the description, traditional displacement estimation approach can be replaced with the proposed method which utilizes different estimation model for constant segment and variational segment separately.

\section{Framework of the proposed approach}

The principle of the proposed approach for pushing displacement estimation is dividing the inertial signal into segments - variational velocity segment (VVS) and constant velocity segment (CVS). The signals beyond these segments should be ignored theoretically. Thus, signals in other segments will be applied with zero velocity update (ZUPT) and errors in current periodic pushing operation will never be transferred to the next, which will clear cumulative errors effectively. Figure 1 shows the framework of the proposed approach. The inertial sensor information is filtered with wavelet-based de-trending and de-noising filter, which help in removing baseline drift and noise, and reveal fluctuation. The result will be used in final estimation based on the proposed MS-KF.

The input signal, filtered angular rate, is processed to remove the influence of gravity on acceleration using the orientation estimation. By combining control chart-based change-point detection and $k$-means clustering, the original inertial data can be segmented and recognized as segments (VVS and CVS). For these segments, displacement can be estimated separately by a proposed cascaded segmental estimation algorithm which contains variational velocity process method (VVM), constant velocity process method (CVM) and ZUPT. Through repeating the periodic processes with corresponding models: 'VVM $\rightarrow$ $\mathrm{CVM} \rightarrow \mathrm{VVM} \rightarrow$ ZUPT', displacement in a period of pushing or advancing can be obtained.

\section{Key techniques}

\section{Wavelet-based de-trending and de-noising}

Before recognizing starting and ending points of VVS, wavelet transformation method is applied to perfectly remove baseline drift and reveal data features. This section introduces notations related to wavelet transformation. Let $\psi(t)$ be the wavelet function, $\phi(t)$ be the scaling function of a multi-resolution analysis $\left\{h_{n}\right\}$ the low-pass filter and $\left\{g_{n}\right\}$ the high-pass filter. The acceleration signal is indicated as $Y=\left\{y_{n}\right\}, n=0,1, \ldots, N-1$; generally, parameter $N=2^{j}$. The scaling function and wavelet function are defined as follows

$$
\begin{aligned}
& \phi_{j, k}(t)=2^{j / 2} \phi\left(2^{-j} t-k\right),(j, k) \in Z^{2}, \\
& \psi_{j, k}(t)=2^{j / 2} \psi\left(2^{-j} t-k\right),(j, k) \in Z^{2} .
\end{aligned}
$$

The wavelet coefficients of $Y$ can be calculated as follows

$$
\begin{aligned}
& s y_{k}^{0}=y_{k}, k=0, \ldots, N-1, \\
& s y_{k}^{j+1}=\sum_{n} g_{n-2 k} s y_{n}^{j}, k=0, \ldots, 2^{-j-1} N, \\
& \mathrm{~d} y_{k}^{j+1}=\sum_{n} h_{n-2 k} s y_{n}^{j} k=0, \ldots, 2^{-j-1} N .
\end{aligned}
$$

The acceleration signal can be reconstructed as

$$
f(x)=\sum_{k} s y_{j_{0}}(k) \phi_{j 0, k}(t)+\sum_{j=j_{0}}^{\infty} \sum_{k} \mathrm{~d} y_{j}(k) \psi_{j, k}(t) .
$$

Since low frequency baseline shift of acceleration significantly increases the estimation error, this study removes low-frequency component that is decomposed at level 8 . Once the acceleration signal is obtained, the de-trended acceleration can be used for further processing. Except the low-frequency component in acceleration, there is some other high-frequency noise. By applying a hard threshold, these components which are decomposed at levels 1 and 2 can be removed from the de-trended signal.

\section{Cascaded segmental estimation algorithm}

The filtered signal can be applied with cascaded segmental estimation algorithm to retrieve displacement. As shown in Figure 5, the proposed algorithm is divided into three cascaded sections; orientation estimation, segment recognition and MS-KF. In order to describe the proposed estimation algorithm, some essential parameters are listed in Table 2 . 


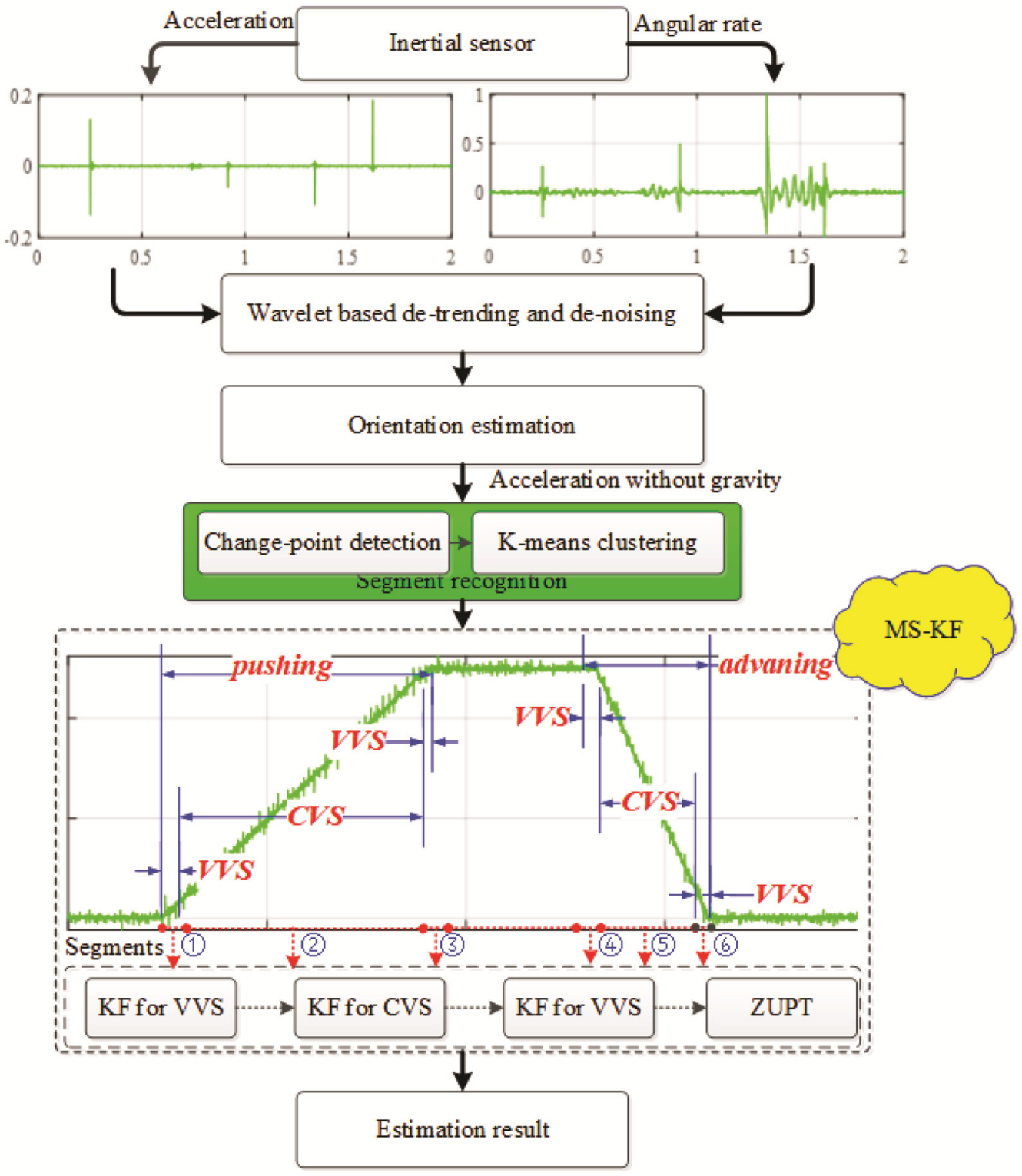

Figure 1. Framework of the proposed approach.

Table 2. Essential parameters

\begin{tabular}{|c|c|c|c|}
\hline Symbol & Representation & Symbol & Representation \\
\hline$L$ & Local (sensor) frame & ${ }^{L} \omega$ & Measured angular rate \\
\hline$G$ & Earth frame & ${ }_{G}^{L} q$ & $\begin{array}{l}\text { Orientation quaternion of the global frame } G \\
\text { relative to the local frame } L\end{array}$ \\
\hline${ }^{L} a$ & Measured acceleration & $\Delta t$ & Sampling time period \\
\hline${ }^{G} a$ & True gravitational acceleration & ${ }^{G} g_{r}$ & True gravity vector ${ }^{G} g_{r}=[0,0, g]^{T}$ \\
\hline $\begin{array}{l}\text { Constant velocity segment } \\
\text { (CVS) }\end{array}$ & Constant velocity segment & $\begin{array}{l}\text { Variational velocity } \\
\text { segment (VVS) }\end{array}$ & Variational velocity segment \\
\hline $\begin{array}{l}\text { Constant velocity process } \\
\text { method (CVM) }\end{array}$ & Constant velocity process model & $\begin{array}{l}\text { Variational velocity } \\
\text { process method (VVM) }\end{array}$ & Variational velocity process model \\
\hline
\end{tabular}

Orientation estimation: Quaternion is widely used in IMU-based MARG system due to the convenient orientation representation. Here we use quaternion-based repre-

CURRENT SCIENCE, VOL. 117, NO. 10, 25 NOVEMBER 2019 sentation to estimate orientation. Angular velocity from the gyroscope is the principle information source, with quaternion transformation and vector integration. The 
predicted orientation quaternion can be described as follows

$$
\begin{aligned}
& { }_{G}^{L} \dot{q}_{w, t}=\frac{1}{2}{ }_{G}^{L} q_{t-1} \otimes{ }^{L} \omega_{t}, \\
& { }_{G}^{L} q_{w, t}={ }_{G}^{L} q_{w, t-1}+{ }_{G}^{L} \dot{q}_{w, t} \Delta t .
\end{aligned}
$$

Using the output of prediction, raw acceleration in local (sensor) frame ${ }^{L} \alpha_{t}$ is rotated into the global frame.

$$
{ }^{G} g^{\prime}=R\left({ }_{G}^{L} q_{w, t}\right)^{L} \alpha_{t}
$$

Theoretically, the results should satisfy the equation

$$
R\left(\Delta q_{\mathrm{acc}}\right){ }^{G} g_{r}={ }^{G} g^{\prime},
$$

where $R(q)$ is the rotation matrix transferred from orientation quaternion, and $q=\left[q_{0}, q_{1}, q_{2}, q_{3}\right]$.

$$
\begin{gathered}
R(q)= \\
{\left[\begin{array}{ccc}
q_{0}^{2}+q_{1}^{2}-q_{2}^{2}-q_{3}^{2} & 2\left(q_{1} q_{2}-q_{0} q_{3}\right) & 2\left(q_{1} q_{3}+q_{0} q_{2}\right) \\
2\left(q_{1} q_{2}+q_{0} q_{3}\right) & q_{0}^{2}-q_{1}^{2}+q_{2}^{2}-q_{3}^{2} & 2\left(q_{2} q_{3}-q_{0} q_{1}\right) \\
2\left(q_{1} q_{3}-q_{0} q_{2}\right) & 2\left(q_{3} q_{2}+q_{0} q_{1}\right) & q_{0}^{2}-q_{1}^{2}-q_{2}^{2}+q_{3}^{2}
\end{array}\right] .}
\end{gathered}
$$

However, there is an algebraic deviation between the predicted results and the true gravity vector. The small deviation is used to correct process error. The deviation quaternion $\Delta \hat{q}_{\text {acc }}$, which is obtained using SLERP-based quaternion interpolation, will be used for later orientation estimation correction. Thus, the final orientation quaternion can be calculated as follows.

$$
{ }_{G}^{L} q_{t}={ }_{G}^{L} q_{w} \otimes \Delta \hat{q}_{\mathrm{acc}} .
$$

Acceleration can be used to calculate velocity and displacement via integration and second integration separately.

$$
\left[\begin{array}{l}
x_{t} \\
v_{t}
\end{array}\right]=\left[\begin{array}{ll}
\int_{0}^{\tau} v_{\tau} & \mathrm{d} \tau \\
\int_{0}^{\tau} G a & \mathrm{~d} \tau
\end{array}\right] .
$$

However, acceleration of rotated inertial sensor is affected by orientation, and produces a differential caused by gravity. In order to tackle this problem, we use the above estimated orientation quaternion to remove the gravity influence on acceleration. The orientation quaternion is used for rotating acceleration from local (sensor) frame of reference to the global reference frame, which yields acceleration without gravity.

$$
{ }^{G} g_{l, t}=R\left({ }_{G}^{L} q_{t}\right)^{L} \alpha_{t}-{ }^{G} g_{r} .
$$

The filtered signal will be segmented, and estimated using MS-KF. During estimation, since the signal in CVS has feature of zero acceleration, CVM will be applied to estimation model. For those segments outside the cycle of 'VVM $\rightarrow \mathrm{CVM} \rightarrow \mathrm{VVM}$ ', the measurement of velocity can be reset to zero, which is famous as zero velocity update (ZUPT), and pushing displacement estimation can be discarded to reduce the drift error.

Segment recognition: For segment recognition we propose a method combining control chart-based changepoint detection and $k$-means clustering method together to recognize VVS and CVS.

Control chart-based change-point detection is an efficient method in automation and industrial quality control, though new approaches based on neuro network ${ }^{30}$, neural fuzzy $^{31}$ and Bayesian ${ }^{32}$ have been proposed in recent years. The present study applies Shewhart individuals control chart to find the mutation points and locations which indicate the sudden changes in acceleration.

In comparison to the rational subgroup control chart, the individuals control chart does not have to wait for the next acceleration sample and speeds up the detection process. The basic schema of Shewhart individuals control chart is detecting location with non-normal probability distribution. For a range of acceleration samples, including $m$ samples, the moving individuals average centre line, upper control limit and lower control limit are obtained from the following

$$
\begin{aligned}
& \bar{y}=\frac{1}{m-1} \sum_{i=2}^{m}\left|y_{i}-y_{i-1}\right|, \\
& \mathrm{UCL}=\bar{y}+\frac{3}{d_{2} \sqrt{n}} \bar{y}, \\
& \mathrm{LCL}=\bar{y}-\frac{3}{d_{2} \sqrt{n}} \bar{y},
\end{aligned}
$$

where $n$ is the sample size and $d_{2}$ is a function of $n$. Data beyond control limits should be recognized as a set of change points and indicated as

$$
P=\left\{p_{i}=y_{i} \mid y_{i} \notin[\mathrm{LCL}, \mathrm{UCL}]\right\}, i=0,1, \ldots, m .
$$

The change-point set contains all the change points which should be applied with Kalman-based estimation to get 
the variational velocity. However, it does not work efficiently for a single discrete acceleration sample. Thus, adjacent change points with small time interval among the cluster members are grouped as a segment. To recognize the different segments, these indexes need to be clustered into several types. K-means clustering method is adopted to effectively solve this problem.

Let $i d x$ denote the index of change points; and $i d x=\left\{i \mid p_{i} \in P\right\}$. Since there are four different segments during a period of movement for PHC, thus $k=4$ and the segment should be indicated as $\left\{s_{k}, k=1,2, \ldots, 4\right\}$. The change-point set can be grouped into $k$ segments by seeking minimal value of objective function by the following two steps

$$
\begin{gathered}
s_{i}^{(t)}=\left\{i d x_{m}:\left\|i d x_{m}-\mu_{i}^{(t)}\right\|^{2} \leq\left\|i d x_{m}-\mu_{j}^{(t)}\right\|^{2},\right. \\
\forall j, 1 \leq j \leq k\}, \\
\mu_{i}^{(t+1)}=\frac{1}{\left|s_{i}^{(t)}\right|} \sum_{i d x_{m} \in s_{i}^{(t)}} i d x_{m},
\end{gathered}
$$

where $\mu_{i}^{(t)}$ is the mean of set $i$ at step $t$. These segments are classified into VVS (where acceleration significantly fluctuates) and CVS (no acceleration change). Acceleration in VVS and CVS will be applied with VVM and CVM respectively, to retrieve the displacement.

MS-KF implementation: For different segments, different state space representations are utilized to eliminate cumulative error when estimating pushing displacement. In the case of VVS, the position $x_{i}$ and velocity $v_{t}$ of the hydraulic support are constituted as state vectors in state space representation.

$$
\left[\begin{array}{c}
x_{e, t} \\
v_{e, t} \\
{ }_{a_{e, t}}
\end{array}\right]=\left[\begin{array}{c}
x_{e, t-1}+v_{e, t-1} \Delta t+\frac{1}{2}{ }^{L} \alpha_{t-1} \Delta t^{2} \\
v_{e, t-1}+\left[R\left({ }_{G}^{L} q_{t}\right)^{L} \alpha_{t-1}-g\right] \Delta t \\
{ }^{L} \alpha_{t-1}
\end{array}\right]+v_{t}
$$

This is a variational velocity model (VVM), especially for VVS, by simple algebra operation. A more general format is obtained as follows

$$
\left[\begin{array}{c}
x_{e, t} \\
v_{e, t} \\
{ }_{{ }_{a_{e, t}}}
\end{array}\right]=\left[\begin{array}{ccc}
I & \Delta t I & I \Delta t^{2} / 2 \\
0 & I & R\left({ }_{G}^{L} q_{t}\right) \Delta t \\
0 & 0 & I
\end{array}\right]\left[\begin{array}{c}
x_{e, t-1} \\
v_{e, t-1} \\
{ }_{a_{e, t-1}}
\end{array}\right]+I \mu_{t}+v_{t} .
$$

With a comparison between eq. (2) and $X_{e, t+1}=F_{t} X_{e, t}+$ $G_{t} \mu_{t}+v_{t}$, parameters are listed as below
$X_{\mathrm{e}, t}$ is the state vector;

$$
X_{e, t}=\left[\begin{array}{c}
x_{e, t} \\
v_{e, t} \\
L a_{e, t}
\end{array}\right] \text {. }
$$

$\mu_{t}$ is the constant control input vector; and

$$
\mu_{t}=\left[\begin{array}{c}
0 \\
-g \Delta t \\
0
\end{array}\right]
$$

$F_{t}$ is the state transformation matrix;

$$
F_{t}=\left[\begin{array}{ccc}
I & \Delta t I & I \Delta t^{2} / 2 \\
0 & I & R\left({ }_{G}^{L} q_{t}\right) \Delta t \\
0 & 0 & I
\end{array}\right]
$$

$G_{t}$ is the control input matrix; here it is an identity matrix. $v_{t}$ is zero mean white process noise with covariance $E\left(v_{t} v_{t}^{\prime}\right)=Q_{t}$.

In our system, to measure the exact distance of push operation, displacement sensor was mounted on the pushing link. The measurement equation is

$$
Z_{t+1}=H_{t} X_{e, t+1}+w_{t+1},
$$

where $H_{t}=\left[\begin{array}{lll}0 & 0 & I\end{array}\right]$ is the observation matrix and $w_{i+1}$ is zero mean white measurement noise with known covariance $E\left(w_{t+1} w_{i+1}^{\prime}\right)=R_{t+1}$. The final estimation can be obtained via recursions as follows

(1) Initialize state: $X_{e, 0}$ and $P_{0}$.

(2) Prior estimate: $X_{e, t+1}^{-}=F_{t} X_{e, t}+G_{t} \mu_{t}$.

(3) Calculate prior mean square error: $P_{t+1}^{-}=F_{t} P_{t} F_{t}^{T}+Q_{t}$.

(4) Calculate Kalman filter gain: $K_{t}=P_{t}^{-} H_{t}^{T}\left(H_{t} P_{t}^{-} H_{t}^{T}+R_{t}\right)^{-1}$.

(5) Posterior estimate:

$$
X_{e, t+1}=X_{e, t+1}^{-}+K_{t+1}\left(Z_{t+1}-H_{t+1} X_{e, t+1}^{-}\right) .
$$

(6) Calculate posterior mean square error:

$$
P_{t+1}=\left(I-K_{t+1} H_{t+1}\right) P_{t+1}^{-} \text {. }
$$

(7) Return back to step (2) until all the observations have been estimated.

The above VVM displacement estimation only works during VVS. For CVS, constant velocity model (CVM) is 


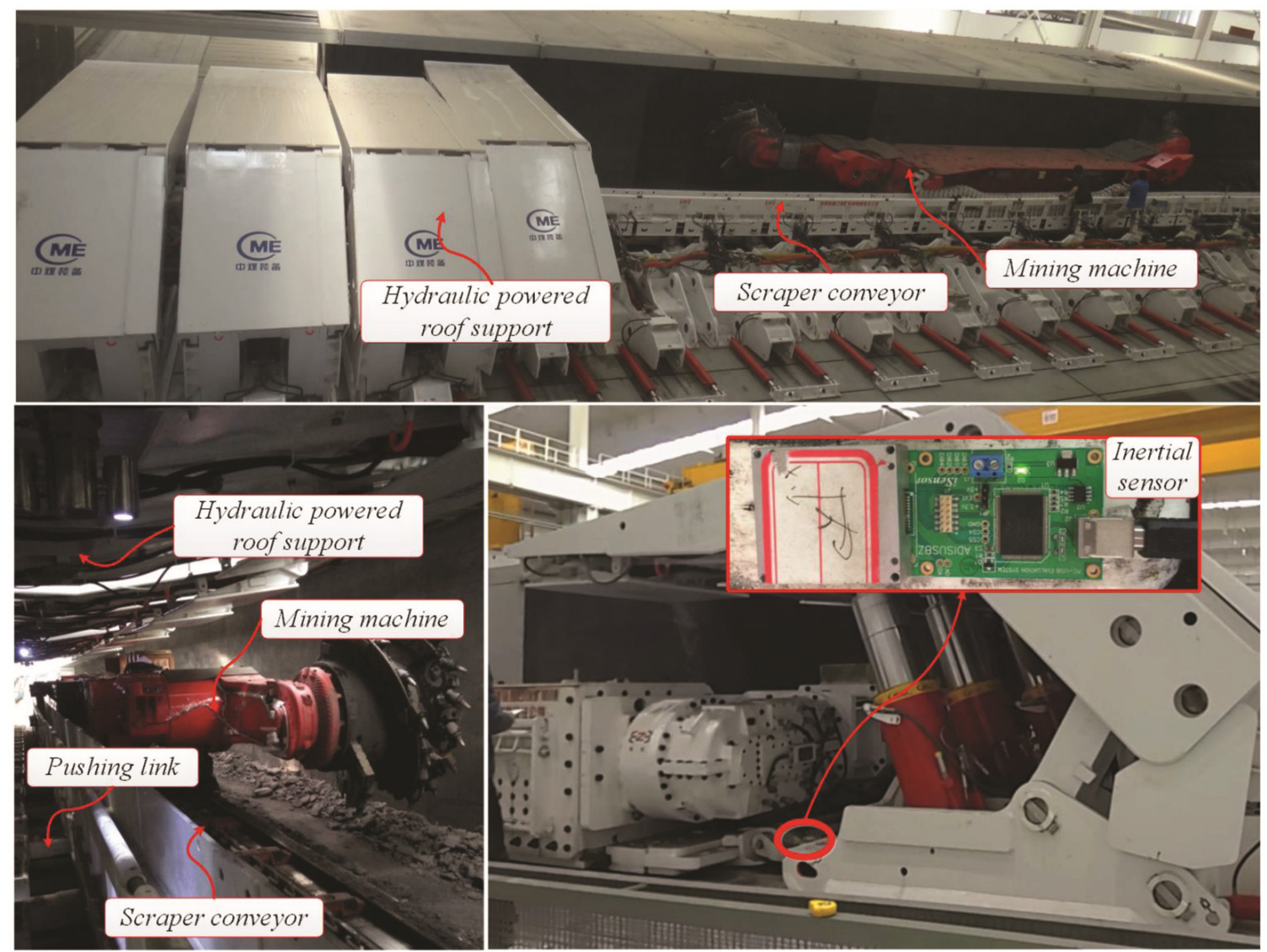

Figure 2. Experimental equipment and layout.

Table 3. Experimental configuration and parameters

\begin{tabular}{lll}
\hline Index & \multicolumn{1}{c}{ Equipment } & \multicolumn{1}{c}{ Information } \\
\hline 1 & Hydraulic support & ZY9000/15/28D \\
2 & Displacement sensor & PR-500 mm \\
3 & Data acquisition card & Smacq USB-4432 \\
4 & IMU sensor & ADIS 16448 \\
\hline
\end{tabular}

applied top process equitation of Kalman filter and is calculated as

$$
X_{i, t+1}=\left[\begin{array}{c}
x_{i, t+1} \\
v_{i, t+1} \\
{ }^{L} a_{i, t+1}
\end{array}\right]=\left[\begin{array}{ccc}
I & \Delta t I & I \Delta t^{2} / 2 \\
0 & I & R\left({ }_{G}^{L} q_{t}\right) \Delta t \\
0 & 0 & 0
\end{array}\right]\left[\begin{array}{c}
x_{i, t} \\
v_{i, t} \\
L a_{i, t}
\end{array}\right]+I \mu_{t}+v_{t} .
$$

For different segments, the initial states are different. Generally, the initial state of VVS when accelerating should be $X_{e, 0}=0, P_{0}=0$, while for segments when decelerating should be $X_{e, 0}=X_{i, t}, P_{0}=0$. Thus, the real displacement is obtained as follows

$$
\begin{gathered}
X_{t}= \\
\begin{cases}X_{e, t} \mid\left\{X_{e, 0}=0, P_{0}=0\right\}, & \operatorname{Mean}\left(\left\{y_{i}\right\}\right)>0, y_{i} \in \mathrm{VVS}, \\
X_{i, t} & y_{i} \in \mathrm{CVS} \\
X_{e, t} \mid\left\{X_{e, 0}=X_{i, t}, P_{0}=0\right\}, & \operatorname{Mean}\left(\left\{y_{i}\right\}\right)<0, y_{i} \in \mathrm{VVS} .\end{cases}
\end{gathered}
$$

\section{Experiment and analysis}

The experiment was conducted using hydraulic support with a PHC. As shown in Figure 2, since the base of hydraulic support remains stationary during pushing operation, the inertial sensor was mounted on the PHC to collect inertial information. In order to obtain the genuine pushing displacement, a high-speed acquisition device and displacement sensor were utilized to collect acceleration data and verify the real pushing displacement.

Table 3 provides the details of the experiment configuration.

\section{Wavelet-based de-trending and de-noising}

Acceleration on $y$-axis is the principle component which contributes to pushing, and it presents distinct acceleration 


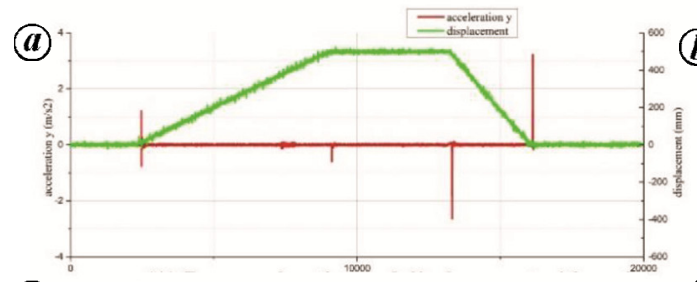

(c)
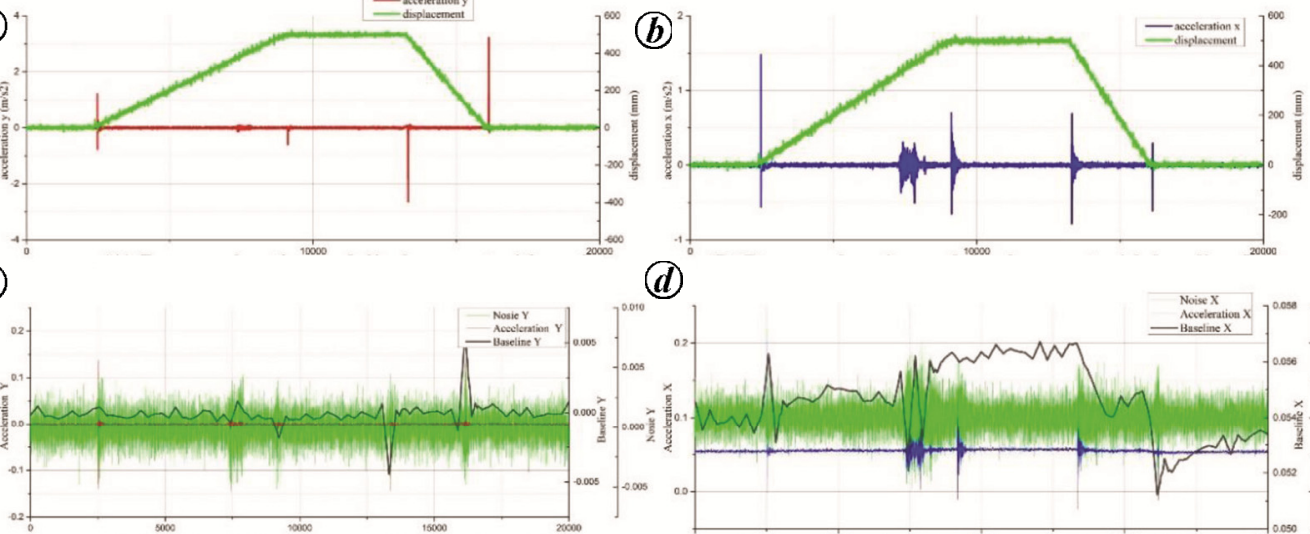

(d)
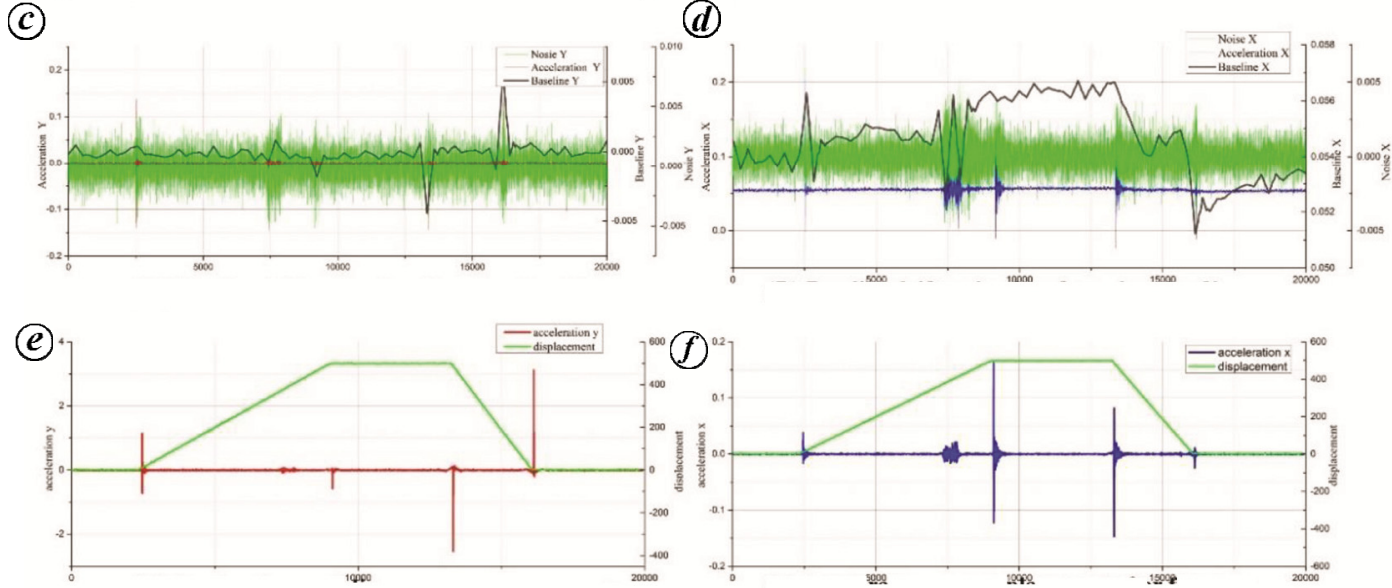

Figure 3. Signal de-trending and de-noising. $\boldsymbol{a}$, Raw acceleration and displacement with noise; $\boldsymbol{b}$, Raw acceleration and displacement with baseline drift; $\boldsymbol{c}$, Baseline drift and noise of acceleration $Y$; $\boldsymbol{d}$, Baseline drift and noise of acceleration $X$; $\boldsymbol{e}$, Acceleration and displacement after filtering; $\boldsymbol{f}$, Acceleration and displacement after filtering.

Table 4. Result of constant speed test and filtering results

\begin{tabular}{lccc}
\hline Segment & $\begin{array}{c}\text { Expected } \\
\text { speed }(\mathrm{mm} / \mathrm{s})\end{array}$ & $\begin{array}{c}\text { Measured } \\
\text { speed }(\mathrm{mm} / \mathrm{s})\end{array}$ & Error (\%) \\
\hline Accelerating & 15.00 & 15.02 & 0.13 \\
Deaccelerating & 35.00 & 35.19 & 0.54 \\
\hline
\end{tabular}

and decelerating features. However, the $x$ - and $y$-axes also represent significant vibration in variational speed segment, as shown in Figure $3 a$ and $b$, which represents acceleration along the $y$-axis and the $x$-axis respectively, and contains high frequency noise and significant baseline drift.

The raw signal was decomposed at level 7 using Coiflet family wavelet; both the high-frequency noise and baseline drift were totally removed. Figure $3 c$ and $d$ shows the removed baseline drift and noise, while Figure $3 e$ and $f$ shows the filtered results. From the result, the magnitudes are found different between extending piston out and withdrawing piston back due to the fact that floating hydraulic cylinder is different. In the experiment, the sampling rate was $1 \mathrm{kHz}$; when extending the piston out, the velocity was $14.5339 \mathrm{~mm} / \mathrm{s}$, while it was $35.1879 \mathrm{~mm} / \mathrm{s}$ when withdrawing the piston back. Table 4 shows details of the results.

\section{Segment recognition results}

Based on the filtered data, Shewhart individuals control chart was applied to find abnormal points in the dataset.
In Figure 4, the recognition of VVS and CVS is represented. The value of acceleration should be limited within the range (LCL, UCL) when accelerating or decelerating. In this experiment, there is an exception; as shown in Figure 4, a vital vibration area occurred before the first deceleration. It should be included in VVS since it affects velocity estimation along the $y$-axis. Thus, the change points are those beyond limitations (Figure $4 a$ ).

Based on these change points, $k$-means clustering method was applied to the processed data with $k=4$, and $k=5$ (Figure $4 b$ and $c$ ). The indices of all the change points clustered into $k$ groups. To process estimation more precisely, $k$ can be increased to a proper value. As shown in this experiment, significant vibration before pushing is stopped and it causes a vital fluctuation of acceleration, thus $k=5$ can be set. The larger the $k$ value set, the more the details available for estimation.

\section{Pushing displacement estimation using MS-KF}

The recognized segments can be applied with different methods to retrieve displacement. As an example, for VVS, let $k=4$. We estimate the segment with variational velocity (e.g. start pushing) and the segment between start pushing and stop pushing with constant velocity (e.g. pushing).

Figure $5 a-c$ shows the estimated acceleration, velocity and displacement respectively. Before pushing, there is a 

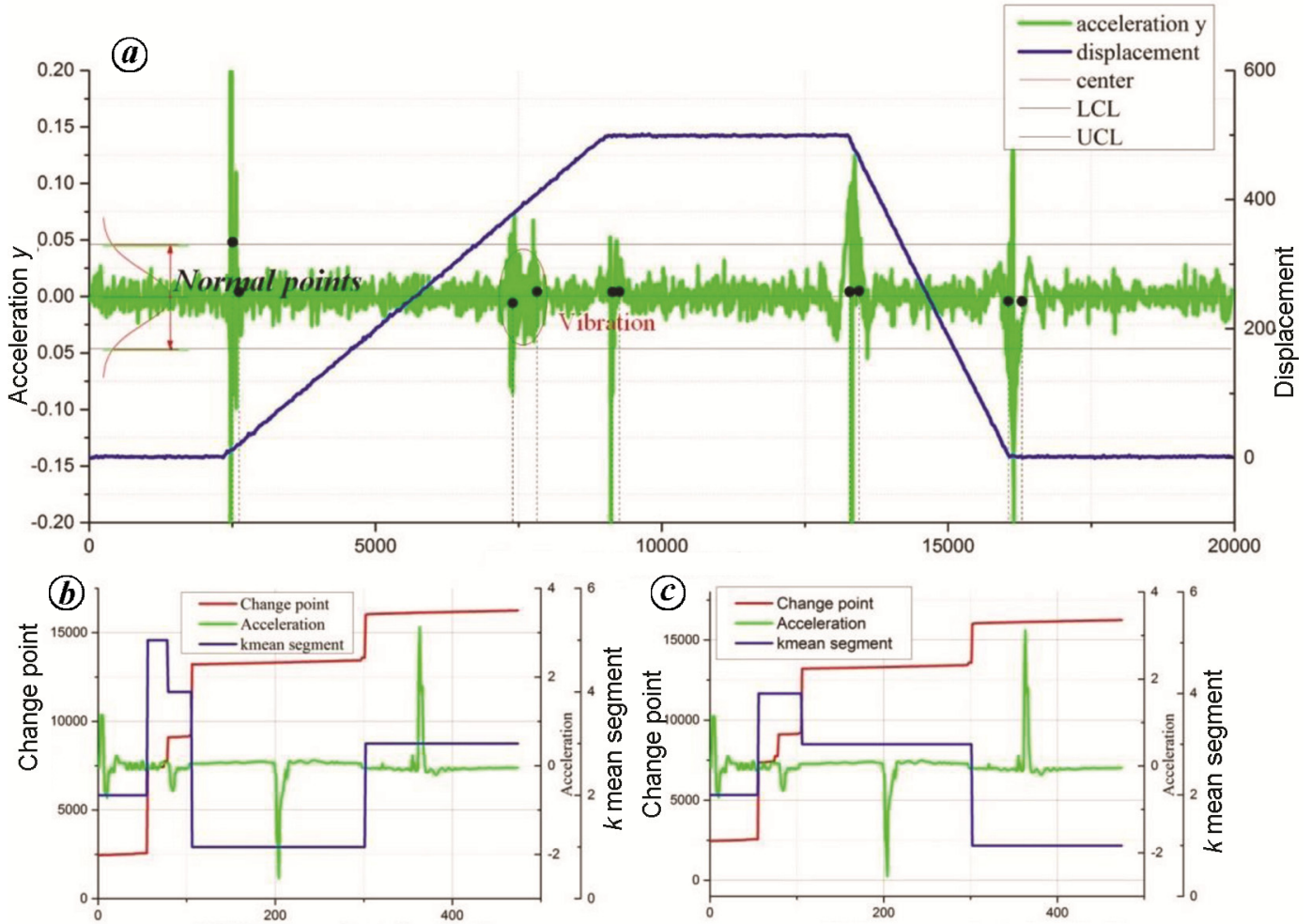

Figure 4. Estimation and integration recognition. $\boldsymbol{a}$, Change-point detection; $\boldsymbol{b}, k$-means clustering $(k=5)$; $\boldsymbol{c}, k$-means clustering $(k=4)$.
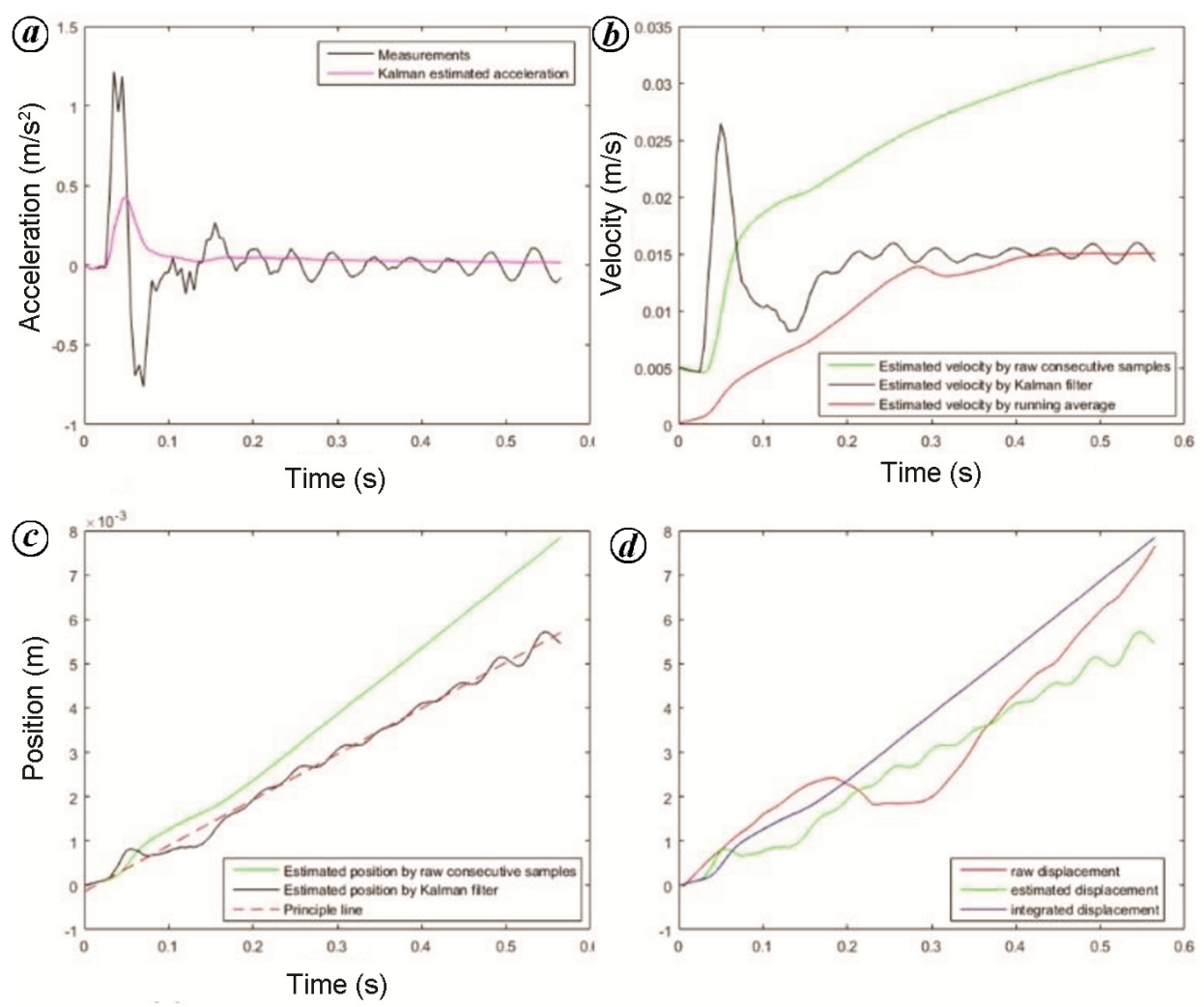

Figure 5. Estimation results (variational velocity). $\boldsymbol{a}$, Estimated acceleration in segment 1 ; $\boldsymbol{b}$, Estimated velocity in segment $1 ; \boldsymbol{c}$, Estimated position in segment $1 ; \boldsymbol{d}$, Estimation error. 

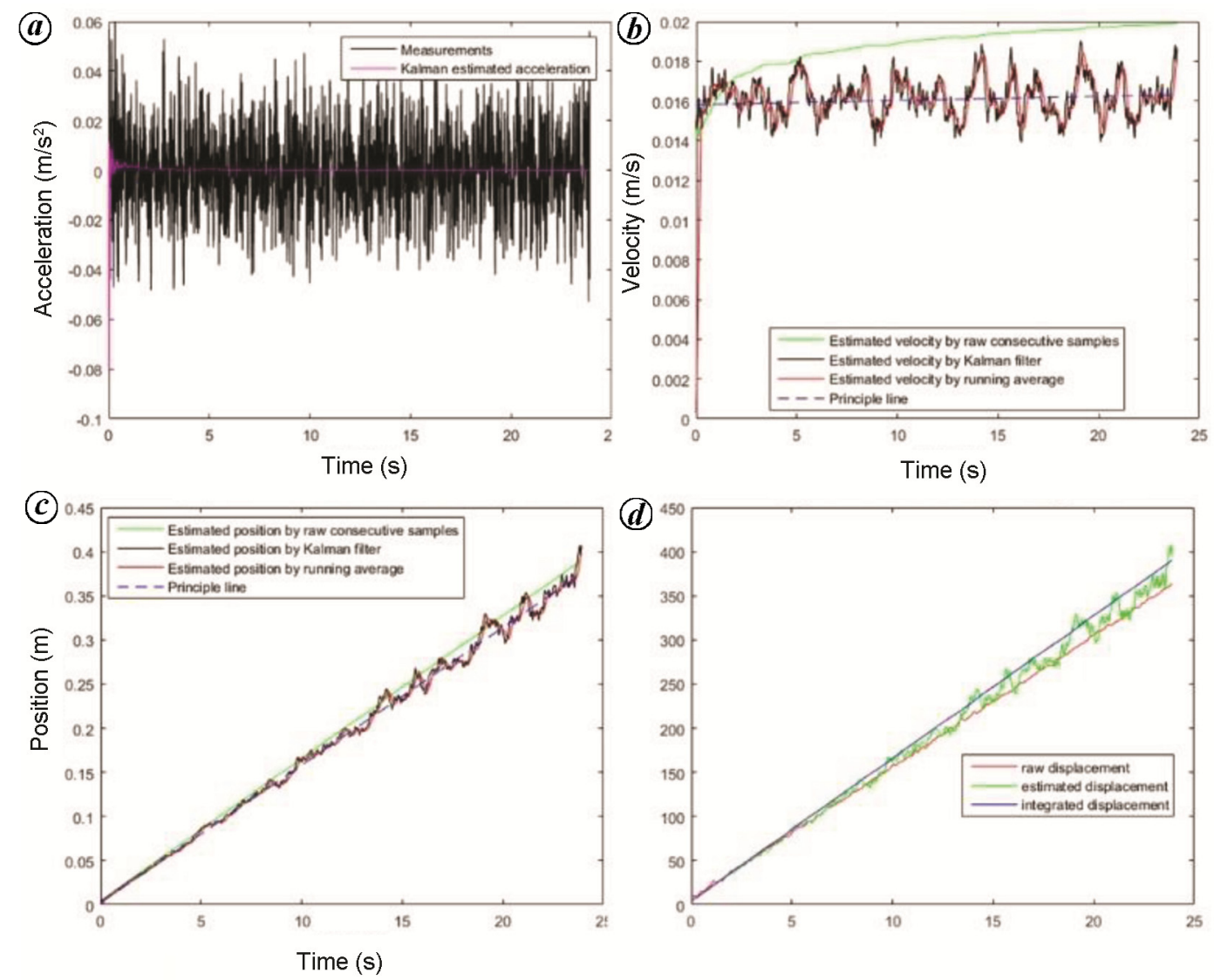

Figure 6. Estimation results (constant velocity). a, Estimated acceleration in segment 2 ; b, Estimated velocity in segment $2 ; \boldsymbol{c}$, Estimated position in segment $2 ; \boldsymbol{d}$, Estimation error.

Table 5. Error analysis result

\begin{tabular}{lcc}
\hline & \multicolumn{2}{c}{ MSE/sec } \\
\cline { 2 - 3 } Segment & Kalman-based estimation & Direct integration \\
\hline VVS & 1.4615 & 1.6859 \\
CVS & 0.445 & 0.637 \\
\hline
\end{tabular}

process of acceleration to a specified velocity which presents a significant acceleration burst (Figure $5 a$ ). Due to elastic characteristics of hydraulic liquid, the acceleration will show a fluctuation between zeros. With Kalman filter, both velocity and displacement are estimated (Figure $5 b$ and $c$ ) respectively. Velocity will be stable after an intense oscillation, with the final velocity of $15.02 \mathrm{~mm} / \mathrm{s}$. However the displacement continues growing to $5.69 \mathrm{~mm}$ approximately. These results should be the initial state for the next estimation segment.

The next estimation state is the pushing segment with constant velocity (CVS) and should be initiated with $\left[y_{0}\right.$, $\left.v_{0}, a_{0}\right]^{T}=[5.69,15.02,0]^{T}$. The estimated acceleration (Figure $6 a$ ), oscillates around zero, which corresponds to the theoretical expectation. The velocity (Figure $6 b$ ) fluc- tuates around a certain value (varies from 14.59 to $18.26 \mathrm{~mm} / \mathrm{s}$, with mean of $16.06 \mathrm{~mm} / \mathrm{s}$ ). The displacement (Figure $6 c$ ), continuously increases to $397.8 \mathrm{~mm}$.

From the two estimation examples, the green lines in Figures 5 and 6 of velocity and displacement estimation represent velocity and displacement integrated directly from raw acceleration respectively. Evidently, both the integrated velocity and displacement show large accumulated error as time increases. The errors are shown in Figures $5 d$ and $6 d$ for these two typical experiments respectively. In order to consider time-based accumulated error, the mean square error per second (MSE/sec) is used for performance comparison (Table 5).

From Table 5, during the same time interval, MS-KFbased estimation for push hydraulic cylinder is better than direct integration, and the accumulated error is no more than $2 \mathrm{~mm} / \mathrm{sec}$ which is capable of monitoring pushing displacement under harsh environments.

\section{Conclusion and future work}

To solve the problem of non-reusable displacement sensor, a cascaded pushing displacement estimation approach has been proposed. In this approach, we utilize a combination 
of wavelet-based de-trending and de-noising filter, change-point detection and $k$-means clustering-based segment recognition and pushing displacement estimation using MS-KF. Examples of the proposed approach have been elaborated, and the results discussed. By applying ZUPT in MS-KF, the cumulative error can be removed before starting the next pushing operation. As a result, the errors are reduced significantly (no more than $2 \mathrm{~mm} / \mathrm{s}$ ) in a periodic pushing operation. Thus the proposed approach is feasible and acceptable in industrial scenario under harsh environments. Theoretically, the proposed MS-KF can be used in other applications with periodic changes. Thus, this approach can be utilized in all scenarios with piston movement.

Compared to traditional measurement method for hydraulic support, this approach provides practical inertial-based displacement estimation. Although the cumulative error is acceptable for hydraulic support, error elimination is still a challenge for more accurate working environment. Our future work will focus on the development of better error elimination algorithms with the help of computational intelligence algorithms, and more comparisons with other intelligent algorithms are necessary. Finally, this approach can be applied in other similar scenarios.

Conflict of interest: The authors declare no conflict of interest.

1. Jeon, J. and Lee, H., Development of displacement estimation method of girder bridges using measured strain signal induced by vehicular loads. Eng. Struct., 2019, 186, 203-215.

2. Pfister, S. T., Kriechbaum, K. L., Roumeliotis, S. I. and Burdick, J. W., Weighted range sensor matching algorithms for mobile robot displacement estimation. In Proceedings 2002 IEEE International Conference on Robotics and Automation, Washington DC, USA, 7 August 2002, pp. 1667-1674.

3. Slimi, T., Moussa, I. M., Kraiem, T. and Mahjoubi, H., Improvement of displacement estimation of breast tissue in ultrasound elastography using the monogenic signal. BioMed. Eng., 2017, 16(1), 19.

4. Yoon, H., Shin, J. and Spencer, B. F., Structural displacement measurement using an unmanned aerial system. Comput.-Aided Civ. Infrastruct. Eng., 2018, 33(3), 183-192.

5. Soman, R., Kyriakides, M., Onoufriou, T. and Ostachowicz, W., Numerical evaluation of multi-metric data fusion based structural health monitoring of long span bridge structures. Struct. Infrastruct. Eng., 2018, 14(6), 673-684.

6. Xu, Y. and Brownjohn, J. M. W., Review of machine-vision based methodologies for displacement measurement in civil structures. J. Civil Struct. Health Monit., 2018, 8, 91-110.

7. Torresan, C. et al., Forestry applications of UAVs in Europe: a review. Int. J. Remote Sensing, 2017, 38(8-10), 2427-2447.

8. Pierzchała, M., Talbot, B. and Astrup, R., Estimating soil displacement from timber extraction trails in steep terrain: application of an unmanned aircraft for 3D modelling. Forests, 2014, 5(6), 1212-1223.

9. Rizzello, G., Naso, D., York, A. and Seelecke, S., Closed loop control of dielectric elastomer actuators based on self-sensing displacement feedback. Smart Mater. Struct., 2016, 25(3), 35034.
10. Rizzello, G., Naso, D., York, A. and Seelecke, S., A self-sensing approach for dielectric elastomer actuators based on online estimation algorithms. IEEE/ASME Trans. Mechatronics, 2017, 22(2), $728-738$.

11. Rizzello, G., Fugaro, F., Naso, D. and Seelecke, S., Simultaneous Self-sensing of displacement and force for soft dielectric elastomer actuators. IEEE Robot. Autom. Lett., 2018, 3(2), 1230-1236.

12. Tessler, A., Roy, R., Esposito, M., Surace, C. and Gherlone, M., Shape sensing of plate and shell structures undergoing large displacements using the inverse finite element method. Shock Vibra., 2018, 2018, 1-8.

13. Golemati, S., Gastounioti, A. and Nikita, K. S., Ultrasound-imagebased cardiovascular tissue motion estimation. IEEE Rev. Biomed. Eng., 2016, 9, 208-218.

14. Mirzaei, M., Asif, A., Fortin, M. and Rivaza, H., Spatio-temporal normalized cross-correlation for estimation of the displacement field in ultrasound elastography, arXiv preprint. 2018, pp. 1804 5305.

15. Pohlman, R. M. et al., Comparison of displacement tracking algorithms for in vivo electrode displacement elastography. Ultrasound Med. Biol., 2019, 45(1), 218-232.

16. Aqel, M. O. A., Marhaban, M. H., Saripan, M. I. and Ismail, N. B., Review of visual odometry: types, approaches, challenges, and applications. SpringerPlus, 2016, 5(1).

17. Ryu, J. H., Gankhuyag, G. and Chong, K. T., Navigation system heading and position accuracy improvement through GPS and INS data fusion. J. Sensors, 2016, 2016.

18. Berrabah, S. A., Sahli, H. and Baudoin, Y., Visual-based simultaneous localization and mapping and global positioning system correction for geo-localization of a mobile robot. Meas. Sci. Technol., 2011, 22, 124003-124003.

19. Olivares, A., Górriz, J. M., Ramírez, J. and Olivares, G., Accurate human limb angle measurement: sensor fusion through Kalman, least mean squares and recursive least-squares adaptive filtering. Meas. Sci. Technol., 2010, 22, 025801-025801.

20. Nam, K., Lee, S., Kuc, T. and Kim, H., Position and velocity estimation for two-inertia system with nonlinear stiffness-based on acceleration sensor. Sensors (Basel, Switzerland), 2015, 16.

21. Cakmak, F., Uslu, E., Yavuz, S., Amasyali, M. F., Balcilar, M. and Altuntas, N., Using range and inertia sensors for trajectory and pose estimation. In Signal Processing and Communications Applications Conference, Trabzon, Turkey, 12 June 2014, pp. 506-509.

22. Gao, J., Webb, P. and Gindy, N., Investigation of an inertialsensor-based dynamic position measurement system for a parallel kinematic machine. Trans. Inst. Meas. Control, 2004, 26, 293 310 .

23. Zhao, H. and Wang, Z., Motion measurement using inertial sensors, ultrasonic sensors, and magnetometers with extended Kalman filter for data fusion. IEEE Sensors J., 2012, 12, 943-953.

24. Coyte, J. L., Stirling, D., Ros, M., Du, H. and Gray, A., Displacement profile estimation using low cost inertial motion sensors with applications to sporting and rehabilitation exercises. In 2013 IEEE/ASME International Conference on Advanced Intelligent Mechatronics: Mechatronics for Human Wellbeing, Wollongong, NSW, Australia, 2013, pp. 1290-1295.

25. Kalman, R. E., A new approach to linear filtering and prediction problems. ASME Trans., J. Basic Eng., 1960, 82, 35-45.

26. Deng, Z. A., Hu, Y., Yu, J. G. and Na, Z. Y., Extended Kalman filter for real time indoor localization by fusing WiFi and smartphone inertial sensors. Micromachines, 2015, 6, 523-543.

27. Urrea, C. and Munoz, R., Joints position estimation of a redundant SCARA robot by means of the unscented Kalman filter and inertial sensors. Asian J. Control, 2016, 18, 481-493.

28. Liu, L. J., Qi, B., Cheng, S. M. and Xi, Z. R., High precision estimation of inertial rotation via the extended Kalman filter. Eur. Phys. J. D, 2015, 69, 1-6. 
29. Qin, F., Chang, L., Jiang, S. and Zha, F., A sequential multiplicative extended Kalman filter for attitude estimation using vector observations. Sensors, 2018, 18(5), 1414.

30. Deng, F., Yang, H. and Wang, L., Adaptive unscented Kalman filter based estimation and filtering for dynamic positioning with model uncertainties. Int. J. Control, Automat. Syst., 2019, 17(3), 667-678.

31. Song, E., Xu, J. and Zhu, Y., Optimal distributed Kalman filtering fusion with singular covariance of filtering errors and measurement noises. IEEE Trans. Autom. Control, 2014, 59(5), 12711282.

32. Zhang, P., Qi, W. and Deng, Z., Parallel covariance intersection fusion optimal Kalman filter. Appl. Mech. Mater., 2014, 475-476.

33. Beravs, T., Begus, S., Podobnik, J. and Munih, M., Magnetometer calibration using Kalman filter covariance matrix for online estimation of magnetic field orientation. IEEE Trans. Instrument. Meas., 2014, 63(8), 2013-2020.

34. Lee, G. B., A fast moving object tracking method by the combination of covariance matrix and Kalman filter algorithm. J. Korea Inst. Inform. Commun. Eng., 2015, 19(6), 1477-1484.

35. Wang, X., You, Z. and Zhao, K., Inertial/celestial-based fuzzy adaptive unscented Kalman filter with covariance intersection algorithm for satellite attitude determination. Aerosp. Sci. Technol., 2016, 48, 214-222.

36. Zhang, L., Wang, Z., Tan, C., Si, L., Liu, X. and Feng, S., A fruit fly-optimized Kalman filter algorithm for pushing distance estimation of a hydraulic powered roof support through tuning covariance. Appl. Sci., 2016, 6(10), 299.

37. Gandomi, A. H. and Alavi, A. H., Krill herd: a new bio-inspired optimization algorithm. Commun. Nonlinear Sci. Numer. Simul., 2012, 17(12), 4831-4845

38. Wang, G., Gandomi, A. H., Alavi, A. H. and Deb, S., A multistage krill herd algorithm for global numerical optimization. Int. J. Artif. Intel. Tools, 2015, 1-17.

39. Wang, G., Deb, S., Gandomi, A. H. and Alavi, A. H., Oppositionbased krill herd algorithm with Cauchy mutation and position clamping. Neurocomputing, 2016, 177(C), 147-157.

40. Wang, G., Gandomi, A. H. and Alavi, A. H., An effective krill herd algorithm with migration operator in biogeography-based optimization. Appl. Math. Model., 2014, 38(9), 2454-2462.

41. Guo, L., Wang, G., Gandomi, A. H., Alavi, A. H. and Duan, H., A new improved krill herd algorithm for global numerical optimization. Neurocomputing, 2014, 138(2), 392-402.
42. Wang, G., Gandomi, A. H., Alavi, A. H. and Deb, S., A hybrid method based on krill herd and quantum-behaved particle swarm optimization. Neural Comput. Appl., 2016, 27(4), 989-1006.

43. Wang, G., Gandomi, A. H., Yang, X. and Alavi, A. H., A new hybrid method based on krill herd and cuckoo search for global optimization tasks. Int. J. Bio-Inspired Comput., 2016, 8(5), 286298.

44. Wang, G., Deb, S. and Cui, Z., Monarch butterfly optimization. Neural Comput. Appl., 2015, 1-20.

45. Wang, G., Deb, S., Zhao, X. and Cui, Z., A new monarch butterfly optimization with an improved crossover operator. Oper. Res., 2016, 1-25.

46. Wang, G., Moth search algorithm: a bio-inspired metaheuristic algorithm for global optimization problems. Memet. Comput., 2018, 10(2), 151-164.

47. Wang, G. G., Deb, S. and Coelho, L. D. S., Earthworm optimization algorithm: a bio-inspired metaheuristic algorithm for global optimization problems. Int. J. Bio-Inspired Comput., 2015, 12(1), $1-22$.

48. Wang, G., Gandomi, A. H., Zhao, X. and Chu, H. C. E., Hybridizing harmony search algorithm with cuckoo search for global numerical optimization. Soft Comput., 2016, 20(1), 273-285.

49. Wang, G., Chu, H. E. and Mirjalili, S., Three-dimensional path planning for UCAV using an improved bat algorithm. Aerosp. Sci. Technol., 2016, 49, 231-238.

50. Wang, G., Guo, L., Duan, H. and Wang, H., A new improved firefly algorithm for global numerical optimization. J. Comput. Theoret. Nanosci., 2014, 11(2), 477-485.

ACKNOWLEDGEMENTS. We thank all the reviewers for their constructive comments. The support of Fundamental Research Funds for the Central Universities (2018B04114), Changzhou Science and Technology Project (No. CE20185040), Project Found by Water Resources Department of Jiangsu Province (2018031), and Changzhou Key Laboratory of Aerial Work Equipment and Intellectual Technology (CLAI201801) in carrying out this work is gratefully acknowledged.

Received 25 April 2019; revised accepted 6 August 2019

doi: $10.18520 / \mathrm{cs} / \mathrm{v} 117 / \mathrm{i} 10 / 1585-1597$ 